\title{
Confidence and Backaction in the Quantum Filter Equation
}

\author{
Wei Cui,, , 冈 Neill Lambert,, , 田 Yukihiro Ota, ${ }^{1}$ Xin-You Lü, ${ }^{1}$ Z. -L. Xiang, ${ }^{1,2}$ J. Q. You, ${ }^{2,3,1}$ and Franco Nori ${ }^{1,4}$ \\ ${ }^{1}$ Advanced Science Institute, RIKEN, Wako-shi, Saitama 351-0198, Japan \\ ${ }^{2}$ Department of Physics, State Key Laboratory of Surface Physics, Fudan University, Shanghai 200433, China \\ ${ }^{3}$ Beijing Computational Science Research Center, Beijing 100084, China \\ ${ }^{4}$ Physics Department, The University of Michigan, Ann Arbor, Michigan 48109-1040, USA
}

(Dated: December 3, 2018)

\begin{abstract}
We study the confidence and backaction of state reconstruction based on a continuous weak measurement and the quantum filter equation. As a physical example we use the traditional model of a double quantum dot being continuously monitored by a quantum point contact. We examine the confidence of the estimate of a state constructed from the measurement record, and the effect of backaction of that measurement on that state. Finally, in the case of general measurements we show that using the relative entropy as a measure of confidence allows us to define the lower bound on the confidence as a type of quantum discord.

PACS numbers: 03.67.-a, 03.65.Ta, 03.65.Yz, 73.63.Kv
\end{abstract}

\section{INTRODUCTION}

Recently there has been a great deal of activity on the topic of "weak" quantum measurements [1-7] in both mesoscopic [8-10] and macroscopic systems [11 14]. In contrast to projective measurement, weak measurements only perturb the system slightly, but in turn can only provide limited information. According to the theory of open quantum systems, both the evolution of the quantum state and its decoherence depend on the systemapparatus coupling strength and the basis in which the measurement system is measured. One of the advantages of such a measurement is that, given a weak continuous measurement record one can reconstruct the quantum system state during its evolution. One particularly interesting approach, which we apply and investigate here, is the "quantum filter equation" as pioneered in the quantum limit by Belavkin [15] and others [16].

The quantum filter equation has been shown to be a powerful method for state reconstruction, and is fairly robust in terms of the resolution needed in describing the measurement record. For example, in recent work [17] it has been shown that the continuous "analogue" measurement record can be reduced to a "one-bit record" and still the filter equation can efficiently produce an estimate (or purification) of the system state. Similarly it has been shown that feedback control can be used to enhance the speed of the state estimation [18 20], and that it can be further optimized when combined with a kind of process tomography 21]. However, successful application of the filter equation requires accurate knowledge of the evolution, both coherent and incoherent, that the monitored system undergoes [22].

Here we first investigate how this state estimation method can be used by considering both how confidently

\footnotetext{
*Electronic address: wcui@riken.jp

${ }^{\dagger}$ Electronic address: nwlambert@riken.jp
}

23] an estimated state deduced from the measurement record reflects the actual state of the system, and how the effect of backaction changes the state. Here the "actual state of the system" means the system under the influence of the back-action [24-26] of the measurement apparatus, not the initial prepared state. In other words, we quantify the confidence of the state-estimation process independently from the overall fidelity of the measurement. Thus, we focus on understanding the state reconstructed using the filter equation, without actively undoing the backaction or employing feedback. We separately define the overall accuracy of the measurement with another quantity or distance, which we term the "epitome". Second, we introduce a new ensemble averaged version of the filter equation which enables a more efficient numerical method (in theoretical treatments) with which to consider state estimation via the filter equation. Third, in the final section we consider the more general situation of an asymptotic positive operator valued measure (POVM), and show that in a limiting case the confidence has a lower bound set by the quantum discord.

Quantities like the confidence have been commonly employed in investigating information gain with projective and general measurements [27, 28]. The behavior of these quantities in the context of weak continuous measurement has not been studied in great detail as of yet, though the concept of information gain and measurement disturbance is well understood [29, 30]. In addition, we point out that our approach here is different from that used in some other works. For example, here we are concerned primarily with the optimal measurement of an unknown state by refining our state of knowledge, whereas in some other approaches the goal is primarily manipulating (or purifying) one's state of knowledge of a given quantum system, and the initial unknown quantum state is unimportant [30].

The model we use here is based on the continuous measurement of a double quantum dot (DQD) charge state using a quantum point contact (QPC) [8, 31 33]. In this situation the tunnelling barrier of the QPC is modulated 
(a)

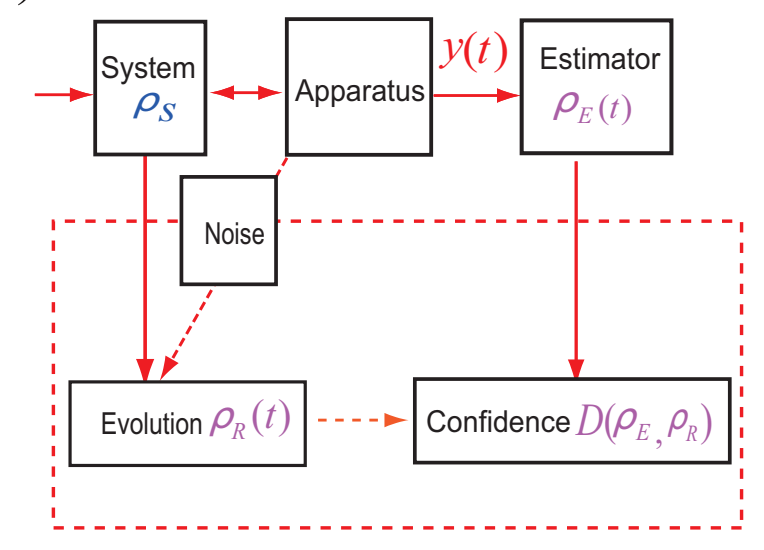

(b)

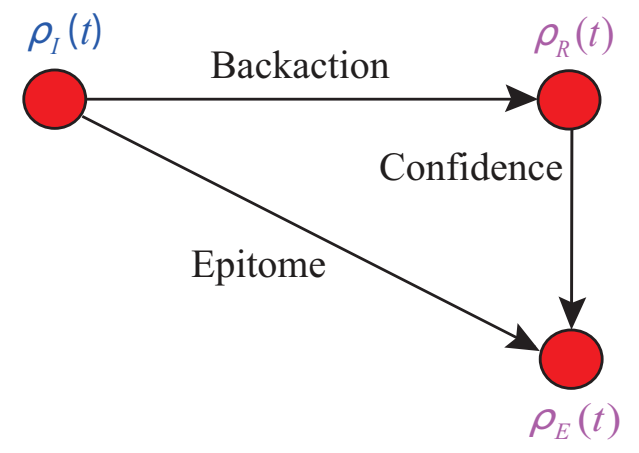

FIG. 1: (Color online) (a) Schematic of the components of the quantum state estimation using the filter equation. Here, $\rho_{s}$ is the system initial state, $\rho_{R}(t)$ is the evolution of the initial state of the system in the measurement-induced noises, $\rho_{E}(t)$ is the estimation of the system state, and $\mathcal{D}\left[\rho_{E}, \rho_{R}\right]$ is the estimation confidence. (b) shows a diagrammatic representation of the confidence $\mathcal{C}$ and backaction $\mathcal{B}$ as the distance between the various states. Note that when we use the relative entropy as a distance measure these quantities are asymmetric (as represented by the one-way arrows).

by the charge in the nearby DQD, and produces a continuous measurement record which can be used in the filter equation. However, the approach is quite general, and recent applications have also arisen in circuit-QED systems [14, 34].

This article is organized as follows: In Sec. II we provide a general definition of the confidence, backaction and epitome. In Sec. III we introduce the model for weak measurement of a DQD, and show the results given by the quantum filter equation. In Sec. IV we speculate about a possible filter equation based on ensemble-averaged measurements. Finally, in Sec. V we show that using the relative entropy as a measure of confidence allows us to define the lower bound on the confidence as the quantum discord.

\section{DEFINITION OF CONFIDENCE AND BACKACTION}

Consider a quantum system $\mathcal{S}$ which interacts with a measurement apparatus $\mathcal{A}$. When implementing a general quantum measurement $\mathcal{M}=\left\{\Pi_{i}\right\}$, our knowledge of the system state is based only on the measured apparatus output $y(t)$. We denote our estimate of the system state as $\rho_{E}(t)$. The initial state of the system is defined as $\rho_{s}(0)$, and $\rho_{R}(t)$ is defined as the evolution of the initial state of the system in the measurement induced noise, i.e., what we can think of as the actual state of the system. We define the ideal state of the system, i.e., its evolution if it were not affected by the measurement apparatus at all, as $\rho_{I}(t)$, i.e., the entirely coherent evolution of $\rho_{s}(0)$.

Definition: The quantum state estimation confidence is defined as

$$
\mathcal{C} \equiv D\left[\rho_{E}, \rho_{R}\right]
$$

where $D[\cdot]$ is some appropriate distance measure. Generally speaking, the smaller $\mathcal{C}$ is the more confident we are of the estimated state, and $\mathcal{C}=0$ if and only if $\rho_{E}=\rho_{R}$, which means that the estimated state is totally confident. Similarly we define the backaction by

$$
\mathcal{B} \equiv D\left[\rho_{I}, \rho_{R}\right]
$$

so that $\mathcal{B}=0$ implies no backaction. Finally, we define the overall accuracy of the measurement with the "epitome",

$$
\mathcal{E} \equiv D\left[\rho_{I}, \rho_{E}\right]
$$

which naturally completes the triangle in Fig. 1. In the treatment that follows of course we have full knowledge of all these states at all times, and can thus in a theoretical sense identify the optimal parameters that minimize these quantities.

There is some freedom in choosing an appropriate measure for $\mathcal{C}, \mathcal{B}$, and $\mathcal{E}$. Here we explicitly consider both the fidelity and the relative entropy 35]. The fidelity is commonly used to measure the effectiveness of the filter equation, and is defined as

$$
F=1-\mathcal{C}=\left|\left(\sigma^{1 / 2} \rho \sigma^{1 / 2}\right)^{1 / 2}\right|^{2} .
$$

We define the confidence as $\mathcal{C}=1-F$ in this case, to match our definition of a distance measure, so that high fidelity implies $\mathcal{C}=0$ (and the same with the other measures). However since the fidelity is a pseudo-distance this lacks some characteristics of a true measure. In the case of the relative entropy we define,

$$
\mathcal{C}=S\left(\rho_{R} \| \rho_{E}\right), \quad \mathcal{B}=S\left(\rho_{I} \| \rho_{R}\right), \quad \mathcal{E}=S\left(\rho_{I} \| \rho_{E}\right)
$$

where

$$
S(\sigma \| \rho)=-\operatorname{Tr} \sigma \ln \rho-S(\sigma)
$$




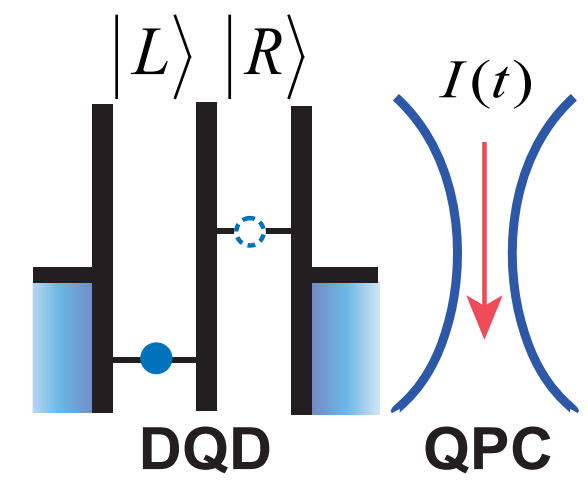

FIG. 2: (Color online) Schematic of a QPC used for measuring the electron states yields backaction on the DQD.

The relative entropy can be seen as a distance measure, though as it is asymmetric in $\sigma \leftrightarrow \rho$ it is technically not. In fact the ordering here is important; with the inverse ordering the backaction $B \rightarrow \infty$ as $\rho_{I}$ is a pure state in this example. Using the relative entropy allows us to make a more direct connection to a general POVM description of a weak continuous measurement in the final section of this work.

\section{CONTINUOUS WEAK MEASUREMENT OF A DOUBLE QUANTUM DOT}

We now present the specific details of the DQD and QPC system. A DQD consists of a dot $L$, connected to an emitter, and dot $R$, connected to a collector [31, 36, 37]. As is typical, we assume that the DQD is in the strong Coulomb regime such that only one electron is allowed in the whole DQD. Here we assume the DQD is prepared in a single electron state, then isolated from the emitter and collector reservoirs via manipulation of gate voltages. The two single-dot states are denoted by $|L\rangle$, and $|R\rangle$. The Hamiltonian of the DQD can be written by

$$
H_{\mathrm{DQD}}=\Omega \sigma_{x} / 2+\epsilon \sigma_{z},
$$

with $\sigma_{x}=|L\rangle\langle R|+| R\rangle\left\langle L\left|, \sigma_{z}=\right| L\right\rangle\langle L|-| R\rangle\langle R|, \epsilon$ is the level splitting between the two single-dot states and $\Omega$ is the coherent tunnelling amplitude betweens the two dots. The nearby $\mathrm{QPC}$ has the Hamiltonian $H_{\mathrm{QPC}}=$ $\sum_{k} \epsilon_{1 k} a_{1 k}^{\dagger} a_{1 k}+\sum_{q} \epsilon_{2 q} a_{2 q}^{\dagger} a_{2 q}$, and the interaction Hamiltonian $H_{I}=\sum_{k, q}\left(\chi-\chi_{L}|L\rangle\left\langle L\left|-\chi_{R}\right| R\right\rangle\langle R|\right)\left(a_{1 k}^{\dagger} a_{2 q}+\right.$ $\left.a_{2 q}^{\dagger} a_{1 k}\right)$, which is modulated by the electron states of the DQD. Here $\chi$ is the tunneling amplitude of an isolated QPC, $\chi_{L}\left(\chi_{R}\right)$ gives the variation in the tunneling amplitude when the extra electron stays on the left (right) dot, and $a_{1 k}\left(a_{2 q}\right)$ denotes the electron annihilation operator for the source (drain) of the QPC. Because the height of the tunneling barrier in the QPC depends on the electron states of the DQD, it is expected that the measured current of the QPC will vary with the DQD states.
In this simple model, the current shot noise [38] of the QPC acts as a noise source. In the low-temperature limit with $k_{B} T \ll \hbar \omega$, the noise spectral density takes the form [37-40.

$J_{I}(\omega)=\frac{4}{R_{K}} D(1-D) \frac{\left(e V_{\mathrm{QPC}}-\hbar \omega\right)}{\left[1-\exp \left\{-\left(e V_{\mathrm{QPC}}-\hbar \omega\right) / k_{B} T\right\}\right]},(8)$

where $R_{k}=h / e^{2}$ is the von Klitzing constant, $D$ is the transparency of a single channel in the QPC, and $D$ is a function of $\chi, \chi_{L}$ and $\chi_{R}$ [9, 39 41]. To treat the measurement signal, or current through the QPC, as a classical variable one must assume that the QPC evolution is much faster than the DQD, so that only the zerofrequency component is important; this is effectively a Markovian limit in terms of treating the QPC backaction on the DQD [8, 24].

We also treat the QPC as a perfect detector. In this limit we can define the measurement strength of the QPC as

$$
\kappa=\frac{(\Delta I)^{2}}{16 J(0)}
$$

Here, $\Delta I=I_{L}-I_{R}$, where $I_{L}=D_{L} e^{2} V / \pi \hbar, I_{R}=$ $D_{R} e^{2} V / \pi \hbar, D_{L}=D+\Delta D, D_{R}=D-\Delta D$, and $\Delta D$ is the change in the transmission of the QPC due to the charge state of the DQD.

The evolution of the real state of the DQD $\rho_{R}$ can be derived using the Bayesian techniques of Korotkov [8 10 ] to give the selective stochastic master equation (SME) in Ito form,

$$
\begin{aligned}
\mathrm{d} \rho_{\mathrm{R}} & =-\frac{i}{\hbar}\left[H_{\mathrm{DQD}}, \rho_{R}\right] d t+\kappa \mathcal{D}\left[\sigma_{z}\right] \rho_{R} d t \\
& +\sqrt{2 \kappa} \mathcal{H}\left[\sigma_{z}\right] \rho_{R} d W_{R},
\end{aligned}
$$

where $\kappa$ is defined above,

$$
\mathcal{H}\left[\sigma_{z}\right] \rho_{R} \equiv \sigma_{z} \rho_{R}+\rho_{R} \sigma_{z}^{\dagger}-\operatorname{Tr}\left(\sigma_{z} \rho_{R}+\rho_{R} \sigma_{z}^{\dagger}\right) \rho_{R},
$$

and the real Wiener process $d W_{R}$ satisfies $\mathrm{E}\left(d W_{R}\right)=$ $0,\left(d W_{R}\right)^{2}=d t$. In Eq. (10), the super-operator $\mathcal{D}$ is defined as

$$
\mathcal{D}[a] \rho=a \rho a^{\dagger}-\frac{1}{2}\left(a^{\dagger} a \rho+\rho a^{\dagger} a\right) .
$$

Given that, in a general sense, the measurement operator is $y=\sqrt{2 \kappa \hbar} \sigma_{z}$ the measurement record increment at a time $t$ is,

$$
\frac{d y(t)}{\sqrt{\hbar}}=\sqrt{8 \kappa}\left\langle\sigma_{z}^{R}(t)\right\rangle d t+d W_{R}=\frac{\Delta I\left\langle\sigma_{z}^{R}(t)\right\rangle}{\sqrt{2 J(0)}} d t+d W_{R} .
$$

Here

$$
\left\langle\sigma_{z}^{R}(t)\right\rangle=\operatorname{Tr}\left[\sigma_{z} \rho_{R}(t)\right]
$$



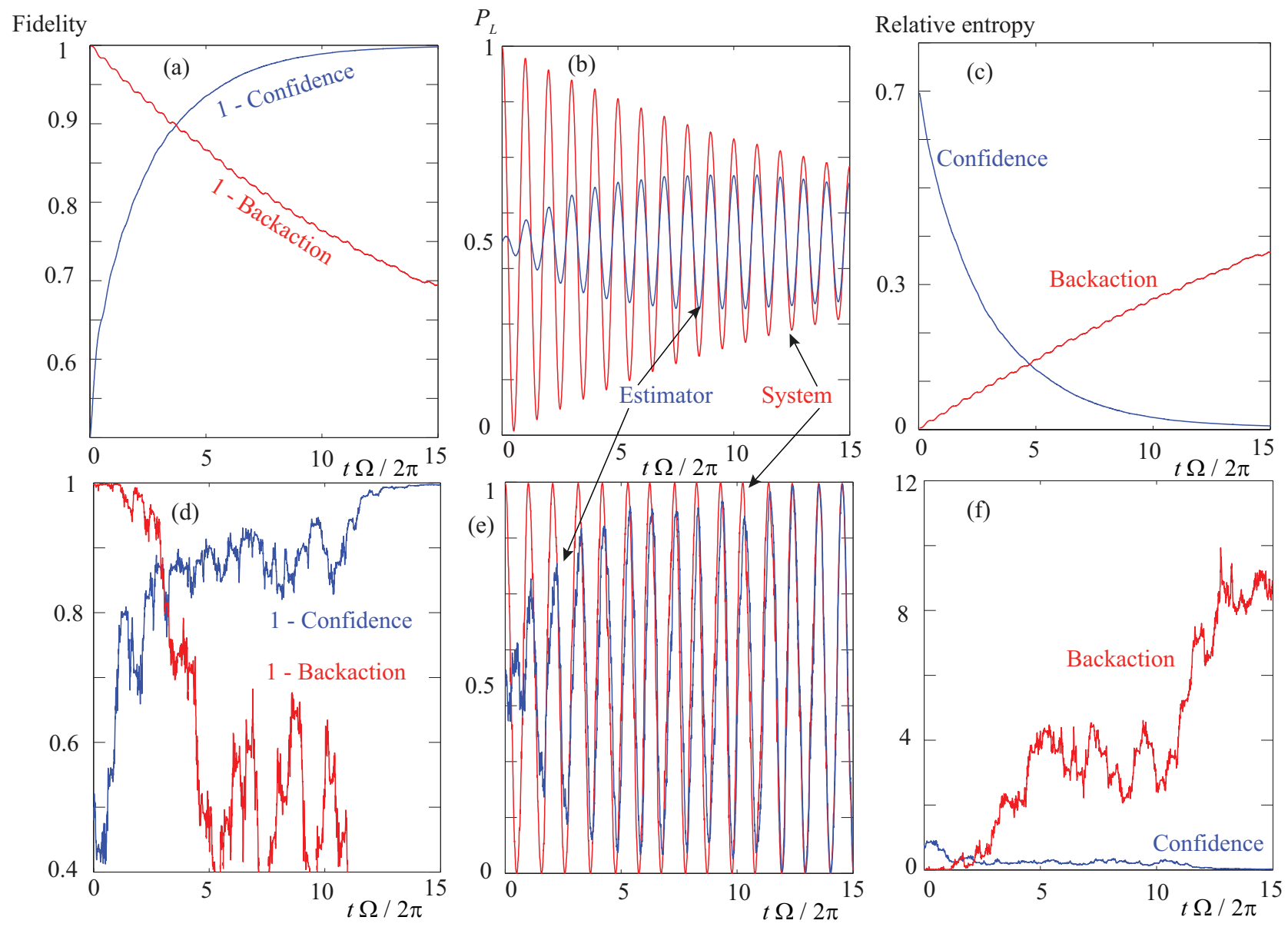

FIG. 3: (Color online). The top row of figures shows the ensemble-averaged behavior (over 2, 000 realizations) of (a) one minus the confidence (blue) and one minus the backaction (red) defined in terms of the fidelity, (b) the occupation of the left "dot" or state for the system, Eq. (10) (in red) and the estimator, Eq. (16), (in blue), and (c) the confidence (blue) and backaction (red) defined in terms of the relative entropy. In all figures $\kappa=0.005 \Omega$, and time is evolved for the equivalent of 15 Rabi oscillations of the bare quantum dot state. Figure (b) shows more directly how the estimator quickly oscillates in phase with the system state, but takes time to evolve to the same population magnitude. The small oscillations seen in both backaction curves in (a) and (c) are typical for the definition of the backaction, and simply represent the "nodes" in the oscillation curve of, e.g., $P_{L}$, where the $\rho_{I}$ and $\rho_{R}$ states coincide. Figures (d)-(e) show the same quantities as (a)-(c) except just a single realization. The estimator state still quickly approaches the system state, as is typical with the filter equation. The backaction changes in magnitude drastically in both (d) and (f). There is no single-realization dephasing in (e) because we assume the QPC to be a perfect detector. This is in contrast to recent work [14] on circuit QED where some information can remain hidden due to the finite lifetime of the measurement cavity. Note, in (a) and (d) we have plotted $1-C$ and $1-B$, so that the fidelity result can be easily compared to other works investigating the effectiveness of the filter equation using fidelity.

is the instantaneous expectation value of $\sigma_{z}$ at time $t$ based on the selectively evolved density matrix $\rho_{R}(t)$.

This equation of motion also relies on the assumption that

$$
\left|I_{L}-I_{R}\right|=|\Delta I| \ll I_{0}=\left(I_{L}+I_{R}\right) / 2
$$

so that many electrons, $N \geq\left(I_{0} / \Delta I\right)^{2} \gg 1$, should pass through the QPC before one can distinguish the quantum state. This is the weakly responding or linear regime, and the model as we have described it is entirely equivalent to the formulation used by Korotkov and others [8-10]. Also, note that for consistency with other works on the filter equation as a state estimation technique [17, 22] we describe the noise as a Wiener process, so that the width of the Gaussian distribution [9, 10] used to describe the weak measurement with a QPC is absorbed into $\kappa$.

\section{A. Quantum Filter Equation}

To estimate the quantum state $\rho_{E}$ from the measurement output we employ the quantum filter equation method [17-20, 42, 43]. The evolution of the estimated state $\rho_{E}$ is described by the following stochastic master equation, identical to the "system" one, except the measurement signal from the system evolution, described 
above, determines the noise term:

$$
\begin{aligned}
& d \rho_{E}=-\frac{i}{\hbar}\left[H_{\mathrm{DQD}}, \rho_{E}\right] d t+\kappa \mathcal{D}\left[\sigma_{z}\right] \rho_{E} d t \\
& +\sqrt{2 \kappa} \mathcal{H}\left[\sigma_{z}\right] \rho_{E}\left[\frac{d y(t)}{\sqrt{\hbar}}-\frac{\Delta I}{\sqrt{2 J(0)}}\left\langle\sigma_{z}^{E}(t)\right\rangle\right],
\end{aligned}
$$

The last term is analogous to the classical innovation in control theory [42, 43], i.e., the difference between the actually measured current and the predicted current with the estimated state. The state estimation process involves setting $\rho_{E}(0)=1 / 2$, and evolving under the noise determined by the measurement record from the "experiment", or in this theoretical work Eq. (10).

As demonstrated in Ref. [22], even a small error in the Hamiltonian of the above equation can induce errors in the estimate of the state provided by the quantum filter equation. We expect the same to be true of the estimates of the noise spectrum of the QPC. Here our goal is to study the efficiency and robustness of quantum state estimation via the filter equation, so as in Ralph et al. 17] we choose the same Hamiltonian and QPC properties in both Eq. (16) and Eq. (10). We leave the problem of accurately estimating the Hamiltonian and the noise properties of the measurement in a dynamic way [22] for future work.

Finally, we combine the time-evolution of these two equations (10) and (16) to calculate the confidence of the estimated state and backaction of the measurement using both the fidelity and the relative entropy, as described earlier. In Fig.3, we show numerical results for these quantities. We will explicitly discuss the epitome in the next section. We set $\rho_{R}(0)=|L\rangle\langle L|, \rho_{E}(0)=1 / 2$, and evolve using the standard techniques for 150000 time steps per Rabi oscillation. The top row of figures shows the results averaged over 2000 realizations, while the bottom row shows just a single realization. In these results we set $\hbar=1, \epsilon=0, \Omega=1$ and $\kappa=0.005 \Omega$. Comparing to real parameters from [37, 40], one could choose a strong inter-dot coupling of the order of $\Omega=32 \mu \mathrm{eV}$, giving a timescale of 130 ps for the Rabi oscillations we show in Fig. 3. This should be chosen carefully however, to match the desired properties of the QPC timescales (or whatever the measurement device happens to be).

When we acquire information from the measurement, it of course induces significant backaction on the system itself. Figure 3.(a) shows the confidence and backaction in terms of the fidelity, while Fig. 3.(c) shows the same in terms of the relative entropy. Both give reasonable descriptions of the distance between the estimated state and system state, and for weak measurement strength the confidence saturates before the backaction does. Obviously then the trade off is to measure on a time scale where both the confidence is relatively high and the backaction is low.

To gain more insight on what is actually happening during the evolution of the filter equation, Fig. 3(b) and
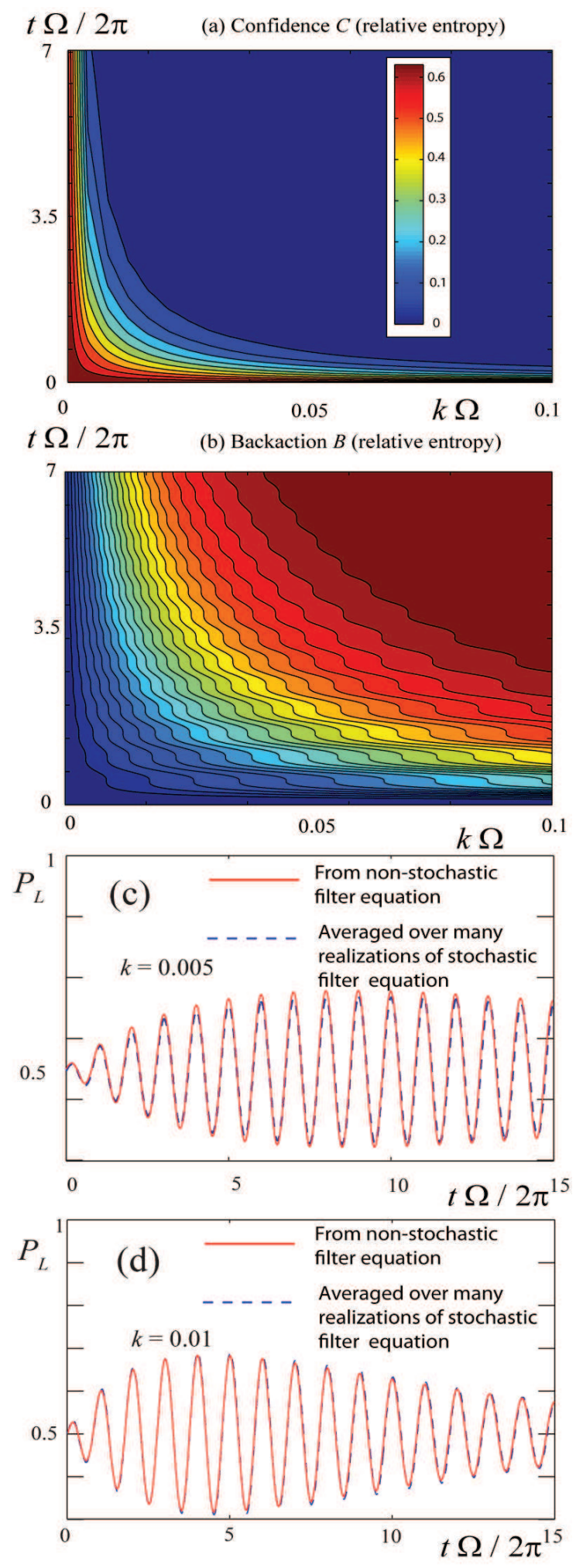

FIG. 4: (Color online) Ensemble-averaged (a) confidence $C$ and (b) backaction $B$ in terms of the relative entropy between system and estimator states, as a function of the interaction time $t$ and measurement strength $\kappa$. Both figures are derived using the ensemble-averaged equation of motion (17). (c) and (d) show a comparison between the probability of occupation of the left $\operatorname{dot} P_{L}(t)$ derived from averaging Eq. 16 over many realizations (dashed blue lines) to that given by solving Eq. [17. 
(e) show the population of the left state of the dot for both the system and estimator. In the ensemble averaged case Fig. 3(b) backaction from the measurement dephases the state, but the estimator matches the system state before coherent information is totally lost. In a single realization, 3(e), the system state does not dephase because the QPC acts as a perfect detector. Compare this to the case of circuit-QED where the lifetime of the cavity can induce dephasing on certain timescales [14, 34]. The off-diagonal elements behave in a similar fashion.

\section{A FILTER EQUATION FOR ENSEMBLE EXPECTATION VALUES}

Solving for the ensemble-averaged results by collating many single realizations is sometimes an arduous numerical task, though can be useful in the stochastic Schrödinger form if one is modelling a system with a large Hilbert space. In practise the system state density matrix ensemble averaged over all measurement trajectories can be trivially calculated by averaging Eq. (10), and noting $E\left[d W_{R}\right]=0$. This gives the expected Lindblad equation of motion which induces the behavior we observe in the backaction. How about the estimator state? Averaging Eq. (16) is non-trivial as the individual trajectories determined by $\left\langle\sigma_{z}^{R}(t)\right\rangle$ are not statistically independent of $\rho_{E}(t)$.

Rather than attempt to do so we simply write down an analogy to the quantum filter equation which depends on ensemble-averaged quantities rather than individual trajectories. We now define $\rho_{E}=\mathrm{E}\left[\rho_{E}\right]$, $\left\langle\sigma_{z}^{R}(t)\right\rangle=\mathrm{E}\left[\left\langle\sigma_{z}^{R}(t)\right\rangle\right]$, and $\left\langle\sigma_{z}^{E}(t)\right\rangle=\mathrm{E}\left[\left\langle\sigma_{z}^{E}(t)\right\rangle\right]$. Thus the term $\mathrm{E}\left[\left\langle\sigma_{z}^{R}(t)\right\rangle\right]$ represents the expectation value extracted from an ensemble averaged version of Eq. (10), i.e., the evolution of the real system under the effect of dephasing. By comparison to the stochastic filter equation we consider the following nonstochastic filter equation,

$$
\begin{aligned}
\frac{\mathrm{d} \rho_{\mathrm{E}}}{\mathrm{dt}} & =-\frac{i}{\hbar}\left[H_{\mathrm{DQD}}, \rho_{E}\right]+\kappa \mathcal{D}\left[\sigma_{z}\right] \rho_{E} \\
& +\sqrt{2 \kappa} \mathcal{H}\left[\sigma_{z}\right] \rho_{E}\left[\frac{\Delta I}{\sqrt{2 J(0)}}\left\{\left\langle\sigma_{z}^{R}(t)\right\rangle-\left\langle\sigma_{z}^{E}(t)\right\rangle\right\}\right] .
\end{aligned}
$$

Solving this equation directly is computationally trivial. To illustrate this we plot the confidence as a function of time and measurement strength $\kappa$ in Fig. 4. We can easily see that as $\kappa$ increases the confidence saturates quickly, but with an associated strong backaction, as expected. Remarkably, if we inspect the density matrix elements of the estimated state generated by Eq. (17) to those generated by the ensemble average over trajectories of Eq. (16) they coincide closely. This is illustrated in Fig. 4(c) and (d). Curiously we are unable to rigorously justify this correspondence, though one can note that Eq. (17) represents a valid solution to Eq. (16) for

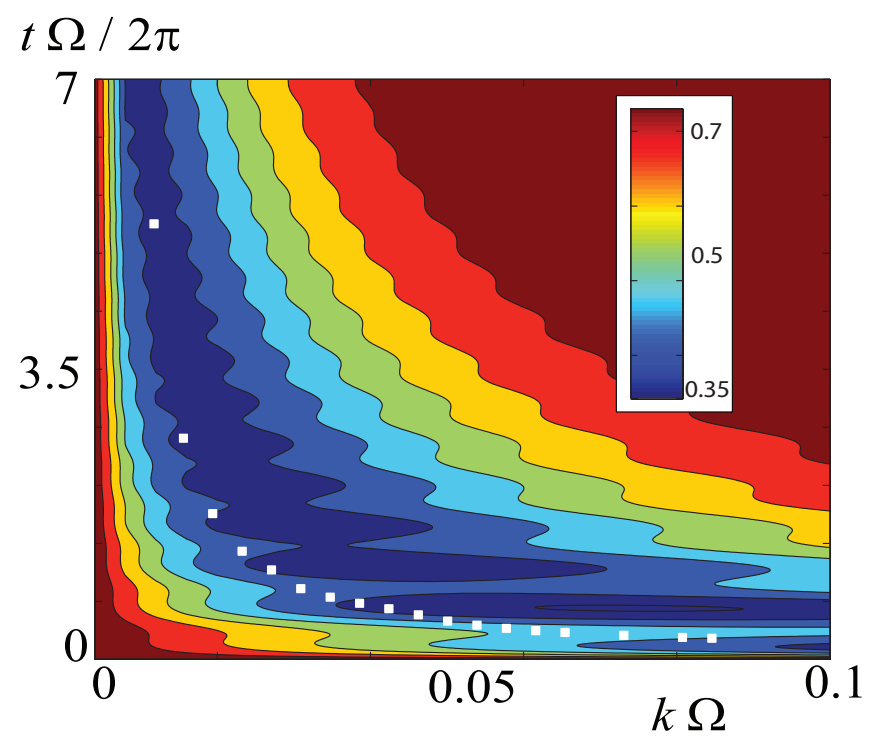

FIG. 5: (Color online) The epitome, $\mathcal{E}=S\left(\rho_{I} \| \rho_{E}\right)$, as a function of the interaction time $t$ and measurement strength $\kappa$. This figure is derived using the ensemble-averaged equation of motion (17). The superimposed white squares indicates the line of crossing points between the confidence and backaction of figure 4, and naturally is close to the minimum in the epitome.

the trajectory determined by $d W_{R}=0$. We also point out that the fictitious nonlinear force in the second line of Eq. (17) is not physical, and the equation may not ensure positivity of the density matrix $\rho_{E}$ at an arbitrary time. Why this works so well in reproducing results from the ensemble averaged filter equation, at least for this case of a single qubit measured in the $\sigma_{z}$ basis, is not clear, and represents a possible avenue of future work.

Finally, in Fig. 5., we use this nonstochastic filter equation to plot the epitome, $\mathcal{E}=S\left(\rho_{I} \| \rho_{E}\right)$. We see that at some intermediate time, depending on the measurement strength, the epitome has an optimal minima which coincides closely to the crossing point of the confidence and backaction. Thus, as one would expect, with continous weak measurements there is an optimal time at which our estimated state is closest to the original, unperturbed, ideal state. In practise this optimization can be also discussed in terms of goal programming (shown in Appendix A) [44]. Further methods to improve the estimation, or minimize backaction, could include feedback or other techniques from quantum control [45].

\section{POVM AND THE DISCORD AS A BOUND ON CONFIDENCE}

In quantum theory one can describe any measurement scenario as a positive-operator valued measure (POVM). For example, weak measurement is sometimes discussed in terms of a POVM on a combined system/measurement-apparatus, where the measurement 
apparatus itself is also considered to be a quantum system. To gain a more fundamental perspective on the confidence and backaction, as we have defined them here, we consider an alternative measurement scheme of an asymptotic POVM.

First, we retain our definition of the initial pure system-apparatus state $\rho_{I}(0)=\rho_{s}(0) \otimes \rho_{A}(0)$. We then assume that a measurement apparatus $A$ is allowed to interact with the system to produce the typically entangled and correlated system-apparatus state $\rho_{s, A}$ (we suppress any time argument here for complete generality). We define the "real" state of the system then as this combined state $\rho_{s, A}$. Finally, we perform measurements on $A$ associated with a POVM $\left\{\Pi_{j}^{A, \dagger} \Pi_{j}^{A}\right\}$, where $\sum_{j} \Pi_{j}^{A, \dagger} \Pi_{j}^{A}=1$. Our estimate of the combined system-apparatus state given by the measurement is $\rho_{m}=\sum_{j} \Pi_{j}^{A} \rho_{R} \Pi_{j}^{A, \dagger}$. Again we define the confidence in terms of the relative entropy, so $C=S\left(\rho_{s, A} \| \rho_{m}\right)$.

The relative-entropy-based confidence has an interesting lower bound in the case when the POVM becomes a projective valued measure. Writing,

$$
C=-\operatorname{Tr} \rho_{s, A} \ln \sum_{j} \Pi_{j}^{A} \rho_{s, A} \Pi_{j}^{A, \dagger}-S\left(\rho_{s, A}\right)
$$

we can substitute $\Pi_{j}^{A}=|j\rangle\langle j|$, where $|j\rangle$ is some distinguishable orthonormal basis describing the measurement apparatus. Then the confidence becomes,

$$
\begin{aligned}
C= & -\operatorname{Tr} \sum_{j}|j\rangle\left\langle j\left|\rho_{s, A} \ln \sum_{j}\right| j\right\rangle\left\langle j\left|\rho_{s, A}\right| j\right\rangle\langle j|-S\left(\rho_{s, A}\right) \\
= & -\operatorname{Tr} \sum_{j}|j\rangle\left\langle j\left|\rho_{s, A}\right| j\right\rangle\left\langle j\left|\ln \sum_{j}\right| j\right\rangle\left\langle j\left|\rho_{s, A}\right| j\right\rangle\langle j| \\
& -S\left(\rho_{s, A}\right)=S\left(\rho_{m}\right)-S\left(\rho_{s, A}\right) \\
\geq & \min _{|\mathrm{j}\rangle}\left[S\left(\rho_{m}\right)\right]-S\left(\rho_{s, A}\right)=\mathcal{D},
\end{aligned}
$$

where $\mathcal{D}$ is the quantum discord [35], when they assume classicality in terms of only one sub-system. In their work the discord has the meaning of the distance between a given state and the closest (system-apparatus) separable state. In other words the lower bound on the confidence is the distance between the real state and the closest separable state, as one would expect with projective measurements.

In the case of a general POVM, one could argue that the minimization of $C$ over all possible POVMs is equivalent to a generalization of the definition of Modi's discord 35]. Finally, we note that there is a correspondence between the estimator state $\rho_{E}$ we discussed in terms of the filter equation, and the partial trace $\operatorname{Tr}_{A}\left(\rho_{m}\right)$ over the state constructed from asymptotic POVM measurements (and the same for the real state $\rho_{R}$ and $\operatorname{Tr}_{A}\left(\rho_{s, A}\right)$ evolved in the measurement noise in the filter equation example).

\section{CONCLUSION}

In many realistic quantum readout architectures the reliability of the quantum measurement output is an important issue. In this article we discussed how to measure the confidence and the backaction of a state reconstructed from continuous weak quantum measurement. As a typical example, we considered a DQD measured by a nearby QPC. Based on the theory of open quantum systems and the quantum filter equation method we briefly discussed the trade-off between measurement confidence and measurement-induced backaction. We also considered a possible filter equation for ensemble averaged results. We finished by discussing the case of a general POVM and how the confidence (when defined as a relative entropy) has a lower bound related to the quantum discord.

\section{Acknowledgments}

We thank Kurt Jacobs for helpful comments. W.C. is supported by the RIKEN FPR Program. Y.O. is partially supported by the Special Postdoctoral Researchers Program, RIKEN. F.N. is partially supported by the ARO, JSPS-RFBR Contract No. 12-02-92100, a Grantin-Aid for Scientific Research (S), MEXT Kakenhi on Quantum Cybernetics, and the JSPS via its FIRST program. J.Q.Y. is partly supported by the National Basic Research Program of China Grant No.2009CB929302, NSFC Grant No.91121015, and MOE Grant No.B06011. X.Y.L. is supported by a JSPS Fellowship.

\section{Appendix A: Quantitative characterization of confidence and backaction via goal programming}

The results in Section IV show a clear trade-off relation between the confidence and the backaction in the parameter space spanned by the interaction time $t$ and the measurement strength $k$. Let us examine this relation via a sophisticated method, goal programming [44]. We formulate our problem setting more specifically; we determine $t$ and $k$ such that $\mathcal{C} \leq \Delta_{C}$ and $\mathcal{B} \leq \Delta_{B}$ for given (small) positive constants $\Delta_{C}$ and $\Delta_{B}$. The two parameters $\Delta_{C}$ and $\Delta_{B}$ are regarded as, respectively, admissible confidence error and permissible backaction. Thus, we can obtain a good measurement scenario to increase the confidence (i.e., minimize $\mathcal{C}$ ) while reducing the backaction (i.e., minimizing $\mathcal{B}$ ). Hereafter, the confidence and the backaction are defined via the relative entropy, as seen in Eq. (5).

Goal programming 44] provides an optimization method to deal with two (or more than two) conflicting objectives and it is widely used in mathematics, information theory and engineering. Instead of finding solutions which absolutely minimize or maximize objective functions, the task of goal programming is to find solutions 
$t \Omega / 2 \pi$
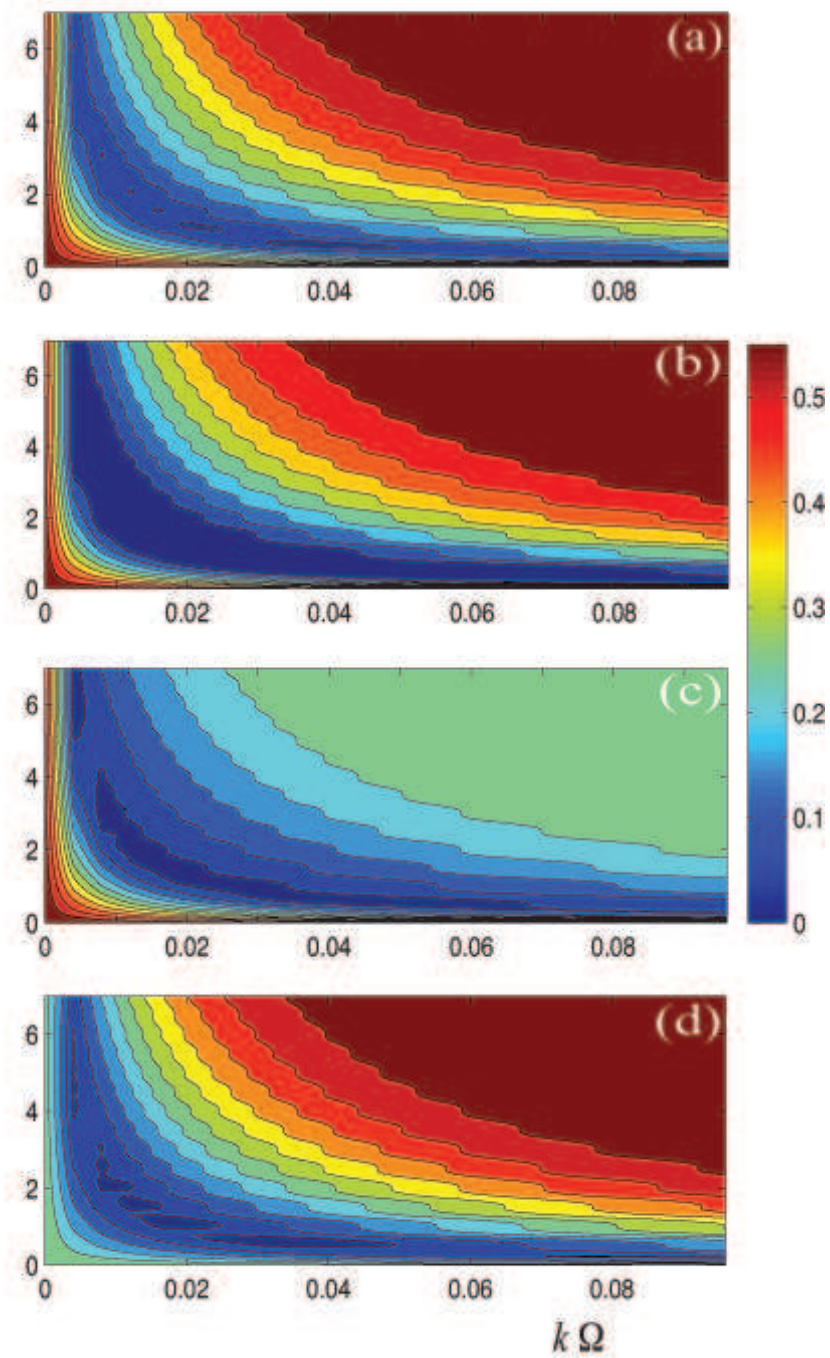

FIG. 6: (Color online) Density profile of the optimization function $\mathcal{O}$ in the goal programming model for several cases: (a) $\eta_{1}=\eta_{2}=1$ and $\Delta_{C}=\Delta_{B}=0.1$; (b) $\eta_{1}=\eta_{2}=1$ and $\Delta_{C}=\Delta_{B}=0.2$; (c) $\eta_{1}=1, \eta_{2}=0.5$ and $\Delta_{C}=\Delta_{B}=0.1$; (d) $\eta_{1}=0.5, \eta_{2}=1$ and $\Delta_{C}=\Delta_{B}=0.1$.

that, if possible, satisfy a set of goals, or otherwise violate the goals minimally. This makes the approach more appealing to practical designers, compared to other optimization methods (e.g., linear programming models).
Let us describe our measurement problem in terms of goal programming:

$$
\begin{array}{ll}
\text { Minimize } & \mathcal{O} \equiv \eta_{1} \delta_{1}^{+}+\eta_{2} \delta_{2}^{+}, \\
\text {subject to } & \left\{\begin{array}{l}
\mathcal{C}(\vec{x})-\delta_{1}^{+}+\delta_{1}^{-}=\Delta_{C} \\
\mathcal{B}(\vec{x})-\delta_{2}^{+}+\delta_{2}^{-}=\Delta_{B} \\
\delta_{1}^{ \pm}, \delta_{2}^{ \pm} \geq 0 \\
\vec{x}=(t \Omega / 2 \pi, k \Omega) \in \mathcal{M}
\end{array}\right.
\end{array}
$$

The weight factors $\eta_{1}$ and $\eta_{2}$ are given positive number, and represent the relative priority of objectives. If $\eta_{1}>$ $\eta_{2}$, the condition for the confidence is prior to the one for the backaction, and vice versa. The condition for the confidence $\left(\mathcal{C} \leq \Delta_{C}\right)$ is reformulated by the relation $\mathcal{C}+\delta_{1}^{+}-\delta_{1}^{-}=\Delta_{C}$, with the deviations between the admissible error and the actual value, $\delta_{1}^{+}$and $\delta_{1}^{-}$. When $\mathcal{C}>\Delta_{C}\left(\mathcal{C} \leq \Delta_{C}\right)$, we set $\delta_{1}^{+}=\mathcal{C}-\Delta_{C}$ and $\delta_{1}^{-}=0$ $\left(\delta_{1}^{+}=0\right.$ and $\left.\delta_{1}^{-}=\Delta_{C}-\mathcal{C}\right)$. Similarly, we can set $\delta_{2}^{ \pm}$via $\mathcal{B}-\delta_{2}^{+}+\delta_{2}^{-}=\Delta_{B}$. The set $\mathcal{M}=\{t \Omega / 2 \pi, k \Omega\}$ is the family of the measurement scenarios. The smaller $\mathcal{O}$, the better performance of the measurement scenario. The minimum value of $\mathcal{O}(\mathcal{O}=0)$ corresponds to the best solution.

Figure 6 shows the contour profile of $\mathcal{O}$ for various cases, based on the ensemble-averaged confidence $\mathcal{C}$ and backaction $\mathcal{B}$. The optimization function $\mathcal{O}$ is a function of the measurement scenarios: the interaction time $t \Omega / 2 \pi$ and measurement strength $k \Omega$. The other parameters are the same as in Figs. 4(a) and (b). In both Figs. 6(a) and (b), the confidence and the backaction have equal importance $\left(\eta_{1}=\eta_{2}=1\right)$. In Fig.6(a) we examine the case that $\Delta_{C}=0.1$ and $\Delta_{B}=0.1$. We find that the best solution $(\mathcal{O}=0)$ appears in the dark blue area. If we relax the restriction to $\Delta_{C}=\Delta_{B}=0.2$, we find that more solutions for the optimization $\mathcal{O}=0$, as seen in Fig. 6(b). We also examine the cases when the confidence has a different importance, or weight, than the backaction, as seen in Figs. 6(c) and (d). The solution for the case where the measurement confidence is more important than the backaction $\left(\eta_{1}=1, \eta_{2}=0.5\right)$ is given in Fig. 6(c). The solution for the opposite situation $\left(\eta_{1}=0.5, \eta_{2}=1\right)$ is given in Fig. 6(d). In the above cases, we have considered a double-criterion goal programming problem and we find that it is convenient for discussing the trade-off relation between measurement confidence and measurement induced backaction.
[1] H. M. Wiseman and G. J. Milburn, Quantum Measurement and Control (Cambridge, New York, 2010).

[2] R. Alicki and M. Fannes, Rep. Math. Phys. 55, 47 (2005).

[3] A. G. Kofman, S. Ashhab, and F. Nori, Phys. Rep. 520.43 (2012).

[4] S. Ashhab, J. Q. You, and F. Nori, Phys. Rev. A 79, 032317 (2009).

[5] S. Ashhab, J. Q. You, and F. Nori, New J. Phys. 11,
083017 (2009).

[6] S. Croke, E. Andersson, S. M. Barnett, C. R. Gilson, and J. Jeffers, Phys. Rev. Lett. 96, 070401 (2006).

[7] V. R. Braginsky and F. Y. Khalili, Quantum measurement (Cambridge, New York, 1992).

[8] A. N. Korotkov, Phys. Rev. B 60, 5737 (1999).

[9] A. N. Korotkov and D. V. Averin, Phys. Rev. B 64, 165310 (2001). 
[10] A. N. Korotkov and A. N. Jordan, Phys. Rev. Lett. 97, 166805 (2006).

[11] I. Buluta, S. Ashhab, F. Nori, Rep. Prog. Phys, 74, 104401 (2011).

[12] J. Q. You and F. Nori, Nature 474, 589 (2011).

[13] J. Q. You and F. Nori, Phys. Today 58 (11), 42 (2005).

[14] A. F. Kockum, L. Tornberg and G. Johansson, Phys. Rev. A. 85, 052318 (2012).

[15] V. P. Belavkin, Rep. Math. Phys. 43 (3), A405 (1999).

[16] H. Carmichael, An Open Systems Approach to Quantum Optics (Springer, New York, 1991).

[17] J. F. Ralph and N. P. Oxtoby, Phys. Rev. Lett. 107, 260503 (2011).

[18] A. C. Doherty, S. Habib, K. Jacobs, H. Mabuchi, and S. M. Tan, Phys. Rev. A 62, 012105 (2000).

[19] B. A. Chase, A. J. Landahl, and J. M. Geremia, Phys. Rev. A 77, 032304 (2008).

[20] J. Zhang, Y-X. Liu, R-B. Wu, C-W. Li, and T-J. Tarn , Phys. Rev. A 82, 022101(2010).

[21] A. Silberfarb, P. S. Jessen, and I. H. Deutsch, Phys. Rev. Lett. 95, 030402 (2005).

[22] J. F. Ralph, K. Jacobs, and C. D. Hill, Phys. Rev. A 84, 052119 (2011).

[23] S. Croke, E. Andersson, S. M. Barnett, C. R. Gilson, and J. Jeffers, Phys. Rev. Lett. 96, 070401(2006).

[24] M. Ozawa, Ann. Phys. (Leipzig) 311, 350 (2004).

[25] F. Buscemi, M. Hayashi, and M. Horodecki, Phys. Rev. Lett. 100, 210504 (2008).

[26] S. Luo and N. Li, Phys. Rev. A 84, 052309 (2011).

[27] K. Banaszek, Phys. Rev. Lett. 86, 1366 (2001).

[28] C. A. Fuchs and A. Peres, Phys. Rev. A 53, 2038 (2001).
[29] A. C. Doherty, K. Jacobs, and G. Jungman, Phys. Rev. A 63, 062306 (2001).

[30] K. Jacobs, Phys. Rev. A 67, 030301 (R) (2003).

[31] S. A. Gurvitz, Phys. Rev. B 56, 15215 (1997).

[32] J. R. Petta et al., Science 309, 2180 (2005).

[33] N. Lambert, C. Emary, Y. N. Chen, and F. Nori, Phys. Rev. Lett. 105, 176801 (2010).

[34] A. N. Korotkov, arXiv:1111.4016v1, (unpublished).

[35] K. Modi, T. Paterek, W. Son, V. Vedral, and M. Williamson, Phys. Rev. Lett. 104, 080501, (2010).

[36] S. H. Ouyang, C. H. Lam, and J. Q. You, Phys. Rev. B 81, 075301 (2010).

[37] K. D. Petersson, J. R. Petta, H. Lu, and A. C. Gossard, Phys. Rev. Lett. 105, 246804 (2010).

[38] R. Aguado and T. Brandes, Phys. Rev. Lett. 92, 206601 (2004).

[39] R. Aguado and L. P. Kouwenhoven, Phys. Rev. Lett. 84, 1986 (2000).

[40] S. Gustavsson, M. Studer, R. Leturcq, T. Ihn, K. Ensslin, D. C. Driscoll and A. C. Gossard, Phys. Rev. Lett. 99, 206804 (2007).

[41] G. B. Lesovik, JETP Lett. 49, 592 (1989).

[42] H. W. Bode, C. E. Shannon, Proc. IRE 38(4), 417-425 (1950).

[43] S. K. Mitter, Nonlinear filtering of diffusion processes, (Springer, New York, 1982).

[44] M. J. Schniederjans, Goal Programming: Methodology and Applications (Kluwer, Boston, 1995).

[45] Y. Watanabe, T. Sagawa, M. Ueda, Phys. Rev. Lett. 104, 020401, (2010). 